\title{
Effects of Charge Transfer in Atomic Bonding on HAADF-STEM Image Simulation
}

\author{
M.L. Odlyzko and K.A. Mkhoyan
}

Chemical Engineering and Materials Science, University of Minnesota, Minneapolis, MN 55455

Conventional implementations of multislice TEM image simulation [1,2] model the electrostatic potential of a solid as that of a collection of unbonded independent atoms; this approximation is known as the "independent atom model" (IAM). It is acknowledged that IAM-based multislice calculations introduce errors in the low-order atomic scattering factor [3], and accordingly several studies have examined the sensitivity of bright-field conventional TEM imaging to bonding orbital charge density, showing that valence charge redistribution due to bonding significantly affects image contrast for thin oxide crystals [4] and atomically thin graphitic crystals [5]. However, no published study has considered the sensitivity of other TEM imaging modes, including high-angle annular-dark-field (HAADF) scanning TEM (STEM), to valence charge distribution.

To begin addressing this gap in understanding, we have employed multislice TEM simulations to examine the effect of valence orbital charge density for HAADF-STEM imaging of the light-element crystals $\mathrm{LiF}, \mathrm{BeO}, \mathrm{BN}, \mathrm{C}, \mathrm{NaF}, \mathrm{MgF}_{2}, \mathrm{MgO}, \mathrm{AlN}$, and $\mathrm{Si}$. The TEMSIM multislice package [6] was used to simulate imaging in a $100 \mathrm{keV}$ aberration-corrected STEM (probe convergence semi-angle 25 mrad, HAADF detector semi-angles 60-300 mrad). Multislice inputs were generated from sample charge densities calculated using the Quantum Espresso package [7]. All materials were modeled at thicknesses up to $50 \mathrm{~nm}$ by IAM, then compared to samples modeled as bonded atoms by density functional theory (DFT) and as independent full-valence-shell ions in the "fully ionized model" (FIM). Images and channeling profiles were simulated along zone axes with each column containing only one type of atom, without thermal vibrations.

As demonstrated by the example of wurtzite AlN aligned to the $(\overline{2} 110)$ zone axis, on-column intensity of the channeling profile (Figure 1), comparisons of raw HAADF linescans (Figure 2), and quantitative comparisons of charge-transfer-inclusive HAADF linescans to IAM linescans (Figure 3) all reflect the sensitivity of HAADF-STEM image simulation to bonding. Results from all crystals studied suggest that clear differences between IAM and charge-transfer-inclusive simulations emerge proportionally to net charge transfer in bonding, being absent in $\mathrm{C}$ and $\mathrm{Si}$ but present in all other materials examined [8].

\section{References}

[1] M.A. O’Keefe et al., Nature 274 (1978), p. 322.

[2] E.J. Kirkland et al., Ultramicroscopy 23 (1987), p. 77.

[3] J.M. Zuo et al., J. Phys. Cond. Matter 9 (1997), p. 7541.

[4] B. Deng and L.D. Marks, Acta Cryst. A 62 (2006), p. 208.

[5] J.C. Meyer et al., Nature Materials 10 (2011), p. 209.

[6] E.J. Kirkland, Advanced Computing in Electron Microscopy, (Springer, New York, 2010).

[7] P. Giannozzi et al., J. Phys. Cond. Matter 29 (2009), p. 395502.

[8] This research was supported by the University of Minnesota Graduate School Fellowship and NSF

DMR-1006706. Drs. M. Cococcioni and B. Himmetoglu are thanked for DFT charge density calculations. 

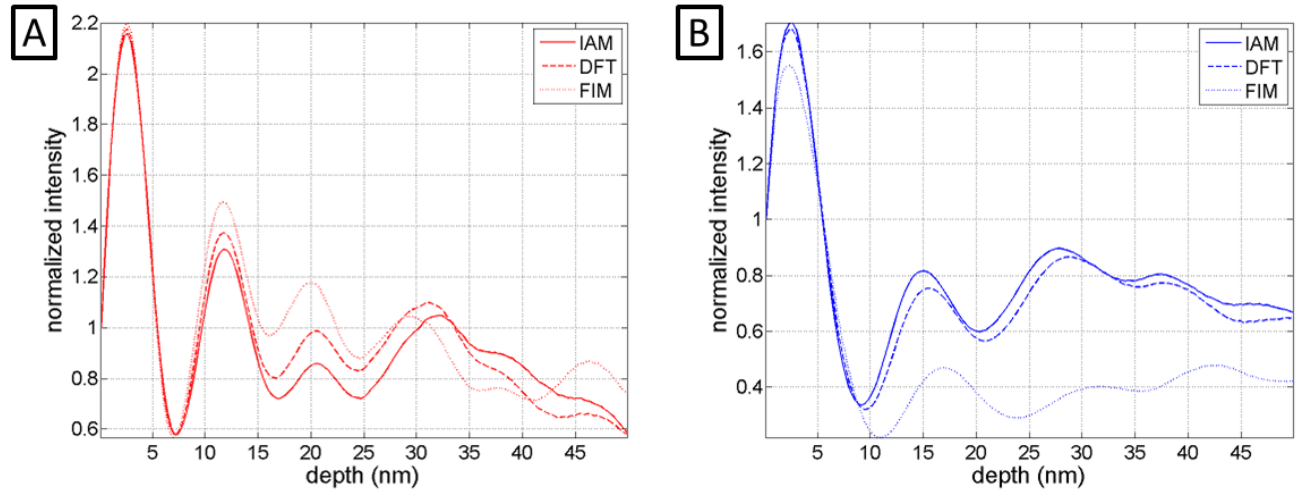

Figure 1. Channeling profiles in $(\overline{2} 110)$-oriented $\mathrm{AlN}$, with probe centered on $(\mathrm{A})$ the $\mathrm{Al}$ column and (B) the $\mathrm{N}$ column. Introducing charge transfer between atoms raises intensity and increases the frequency of intensity oscillations for the net-positively-charged Al column, while decreasing the intensity and decreasing the oscillation frequency for the net-negatively-charged $\mathrm{N}$ column.
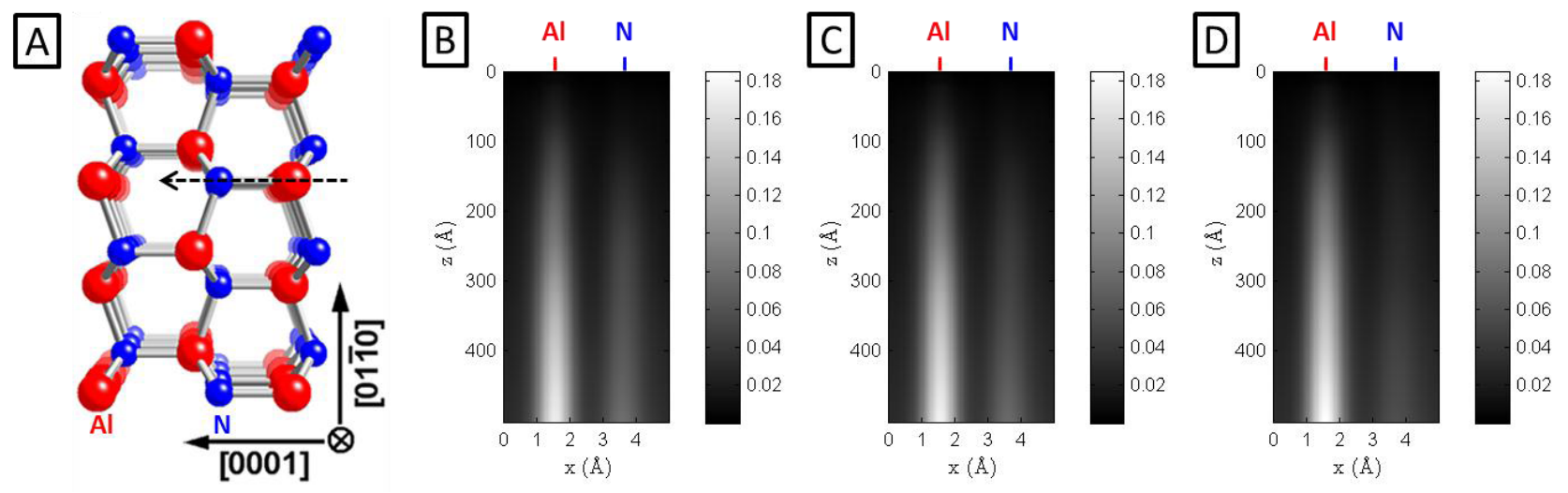

Figure 2. (A) Model of ( $\overline{2} 110)$-oriented AlN, with linescan range indicated by dotted arrow. Profiles of the HAADF linescan as a function of depth for (B) IAM, (C) DFT, and (D) FIM cases are plotted in units of normalized probe current. As net negative charge transfer from Al to $\mathrm{N}$ increases, the Al column intensity increases and the $\mathrm{N}$ column intensity decreases, as expected from the channeling profiles.
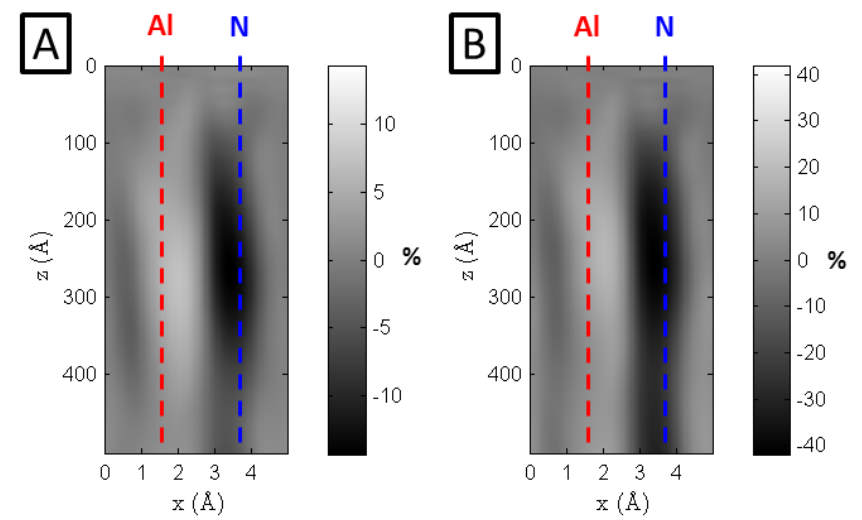

Figure 3. Difference between HAADF image simulations of ( $\overline{2} 110)$-oriented AIN for (A) IAM and DFT inputs and (B) IAM and FIM inputs, normalized to the IAM intensity at each pixel. For both DFT and FIM cases, beyond a depth of about $10 \mathrm{~nm}$ the Al column intensity increases relative to IAM and the $\mathrm{N}$ column intensity weakens, albeit more strongly in the FIM case. 